\title{
Improving Medical Education: A Narrative Review
}

Review began 10/10/2021
Review ended 10/11/2021
Published 10/14/2021
@ Copyright 2021
Joshi et al. This is an open access article
distributed under the terms of the Creative
Commons Attribution License CC-BY 4.0.,
which permits unrestricted use, distribution
and reproduction in any medium, provided
the original author and source are credited.
Tilak Joshi ${ }^{1}$, Pravash Budhathoki ${ }^{2}$, Anurag Adhikari ${ }^{3}$, Ayusha Poudel ${ }^{4}$, Sumit Raut ${ }^{5}$, Dhan B. Shrestha ${ }^{1}$

1. Department of Internal Medicine, Mount Sinai Hospital, Chicago, USA 2. Department of Internal Medicine, BronxCare Health System, Bronx, USA 3. Intensive Care Unit, Nepal Korea Friendship Municipality Hospital, Madhyapur Thimi, NPL 4. Department of Emergency Medicine, Alka Hospital Pvt. Ltd., Kathmandu, NPL 5. Department of Internal Medicine, Kathmandu Medical College, Kathmandu, NPL

Corresponding author: Dhan B. Shrestha, medhan75@gmail.com

\begin{abstract}
With the advancement in basic and clinical sciences, medical education is also constantly evolving. The Accreditation Council for Graduate Medical Education (ACGME) has endorsed six core competencies to improve teaching and learning. This narrative review was conducted after searching the article databases (PubMed, PubMed Central, Embase, and Scopus) about the core competencies such as medical knowledge (problem-based learning), interpersonal communication, patient care, professionalism, practice-based learning and improvement, and system-based care endorsed by ACGME. We included randomized and quasiexperimental trials, cohorts, and case-control studies in this narrative review. In a problem-based learning modality, a real-life scenario is allocated to a group of students. Studies have shown that it is more effectively demonstrated by a better post-test score, improved concentration, and application of knowledge. Interpersonal communication skills promote collaboration with interdisciplinary teams, work quality, and patient adherence to treatment. Professionalism is a human attribute that creates a pleasant work environment and is an essential trait that improves patients' adherence to treatment. In system-based care, patients are benefitted through a well-structured plan of care. Finally, in practice-based learning, medical trainees learn to systematically evaluate the pattern of care and practice the best modality to improve the overall patient care and physician satisfaction. These core competencies need to be incorporated into all levels of medical training.
\end{abstract}

Categories: Internal Medicine, Medical Education, Quality Improvement

Keywords: students, physicians, patient care, communication, medical education, professionalism, problem-based learning

\section{Introduction And Background}

Medical education is ever-evolving because of constant advancements in basic sciences, clinical skills, and professionalism. As a result, it has changed drastically over the last two decades [1]. In 1999, the Accreditation Council for Graduate Medical Education (ACGME) endorsed a set of competencies required in every practicing physician. The six ACGME core competencies are professionalism, patient care, medical knowledge, interpersonal and communication skills, system-based care, and practice-based learning and improvement. Recently, the ACGME has developed the milestones as the roadmap of growth and development based on the core competencies [2]. We intend to provide an up-to-date narrative review in each core competency.

Medical knowledge acquisition and retention is a challenge that the traditional lecture-based teaching method has not addressed. The problem-based learning (PBL) approach was first designed and carried out at McMaster University in Hamilton, Ontario, Canada, in 1969. Its main goal was to promote active learning among students through self-directed and group-based learning. This method stood ahead of the traditional concept of a teacher as the primary educator. The PBL format is widely used in medical schools, where students are allocated into small groups and are provided with cases that resemble real-life scenarios. Students are involved in self-directed learning followed by group discussions, which a tutor accompanies [3].

Interpersonal communication is a vital part of medical education, where health professionals from various departments collaborate to enhance the learning curve and promote patients' overall health status. Incorporating interpersonal communication in medical education is vital for preparing trainees to work in interdisciplinary teams [4]. Patient care is a broad term but essential to consider while acquiring medical education and clinical skills. For example, the interdisciplinary multimodal pain rehabilitation (IMPR) approach collaborates among health professionals involved in patients' physical and psychosocial wellness and educating them about their diseases and treatment strategies. Subsequently, patient care is fostered with this systematic and meticulous approach [5].

Professionalism is a critical clinical discipline that every health personnel must follow from the beginning of their medical career. The belief that personality development is primarily an intrapersonal phenomenon supports the idea of professionalism as a collection of human traits and mutable attributes [6]. The Association of American Medical Colleges (AAMC) in 1991 has provided a set of examples that describe 
medical professionalism, which fellow health professionals have used to gauge their professionalism in medical education [7]. However, different institutions have different ways of assessing it.

Although several evidence-based care treatment plans are developed for diverse medical conditions, they cannot be executed without well-functioning multidisciplinary teams. A system-based care strategy involves planning, cooperation, and a structured management plan based on evidence that benefits patient care [8].

In practice-based learning and improvement, the trainees in medicine are expected to systematically investigate and evaluate their care to their patients and the practice patterns of their workplaces to identify the areas of improvement. They incorporate the best practices and implement the changes with the goal of improvement. They participate in educating patients, families, students, and other healthcare professionals [9].

All these sectors have a crucial effect on the upliftment of medical education.

\section{Review}

\section{Methods}

We searched different databases such as PubMed, PubMed Central, Embase, and Scopus. Studies regarding problem-based learning, interpersonal communication, patient care, professionalism, practice-based learning and improvement, and system-based care were used for this review. Here, we have the narrative review to comply with the above terms.

\section{Findings}

Problem-Based Learning

Nine studies included in our problem-based learning review revealed its tremendous benefits. Most studies assessed this by conducting pre- and post-PBL sessions and pre- and post-traditional lecture-based sessions. Studies showed an increase in post-test scores after PBL sessions in comparison to team-based learning (TBL) $[4,10]$. This is further supported by the meta-analysis findings of Qin et al. and Galvao et al. [11,12].

In contrast, a study done in Harvard Medical School demonstrated no significant difference among the students whose mean prior exam scores were above the median in either group [7]. Apart from the scores, PBL improved attention, application of knowledge, better use of time, and available resources compared to conventional forms of learning $[1,4,5,11,12]$. Likewise, $97 \%$ of the students strongly agreed that PBL sessions should be part of the normal curriculum [11]. Due to the effect of the current pandemic, web-based learning has also become popular among medical students. Virtual learning was found to be as effective as face-to-face interaction learning [13].

Among nine studies on problem-based learning, four were from the USA, three from Europe, and one each from Africa and India. Five studies were from the medical profession, one each from nursing, dental, and physician assistant. Six studies enrolled undergraduate level, two including residents, and one enrolling postgraduate level. Three studies were randomized controlled trials (RCTs), two each cohort, a pre- and post-test single-group study, one each quasi-experimental trial, and a case-control study. A summary of some of the studies on problem-based learning is mentioned in Table 1 [10,13-20].

\begin{tabular}{|c|c|c|c|c|c|}
\hline $\begin{array}{l}\text { S. } \\
\text { No. }\end{array}$ & Study ID & Country & Experimental (PBL/TBL) & Control (Traditional learning) & $\begin{array}{l}\text { P- } \\
\text { value }\end{array}$ \\
\hline \multirow{2}{*}{1} & \multirow{2}{*}{$\begin{array}{l}\text { Alaagib et al. } \\
\text { (2018) [14] }\end{array}$} & \multirow{2}{*}{ Sudan } & $\begin{array}{l}\text { Physiology: } 7.95 \pm 1.65 / 10 \text { (Mean } \pm \text { SD/ Total marks) }(\mathrm{N}= \\
\text { 101) }\end{array}$ & $\begin{array}{l}\text { Physiology: } 9.68 \pm 2.59 / 10 \text { (Mean } \pm \mathrm{SD} / \text { Total } \\
\text { marks) }(\mathrm{N}=146)\end{array}$ & $\begin{array}{l}p< \\
0.001\end{array}$ \\
\hline & & & $\begin{array}{l}\text { Respiratory: } 9.68 \pm 2.59 / 20 \text { (Mean } \pm \text { SD/Total marks) }(\mathrm{N} \\
=146)\end{array}$ & $\begin{array}{l}\text { Respiratory: } 8.60 \pm 4.02 / 10(\mathrm{~N}=146) \text { (Mean } \\
\pm \mathrm{SD} / \text { Total marks) }\end{array}$ & $\begin{array}{l}p= \\
0.006\end{array}$ \\
\hline \multirow{4}{*}{2} & \multirow{4}{*}{$\begin{array}{l}\text { Robson et al. } \\
\text { (2009) [13] }\end{array}$} & \multirow{4}{*}{$\begin{array}{l}\text { United } \\
\text { Kingdom }\end{array}$} & $\begin{array}{l}\text { Mean score improvement in extended matching questions } \\
\text { (EMQs) (E-PBL) ( } N=\text { total students) }\end{array}$ & \multirow{4}{*}{ Not applicable } & \\
\hline & & & CKD = $0.009(\mathrm{~N}=23)$ & & $\begin{array}{l}\mathrm{p}= \\
0.998\end{array}$ \\
\hline & & & $C D=20.6(N=17)$ & & $\begin{array}{l}p= \\
0.014\end{array}$ \\
\hline & & & $\mathrm{UTI}=12(\mathrm{~N}=18)$ & & $\begin{array}{l}p= \\
0.062\end{array}$ \\
\hline
\end{tabular}




\section{Cureus}

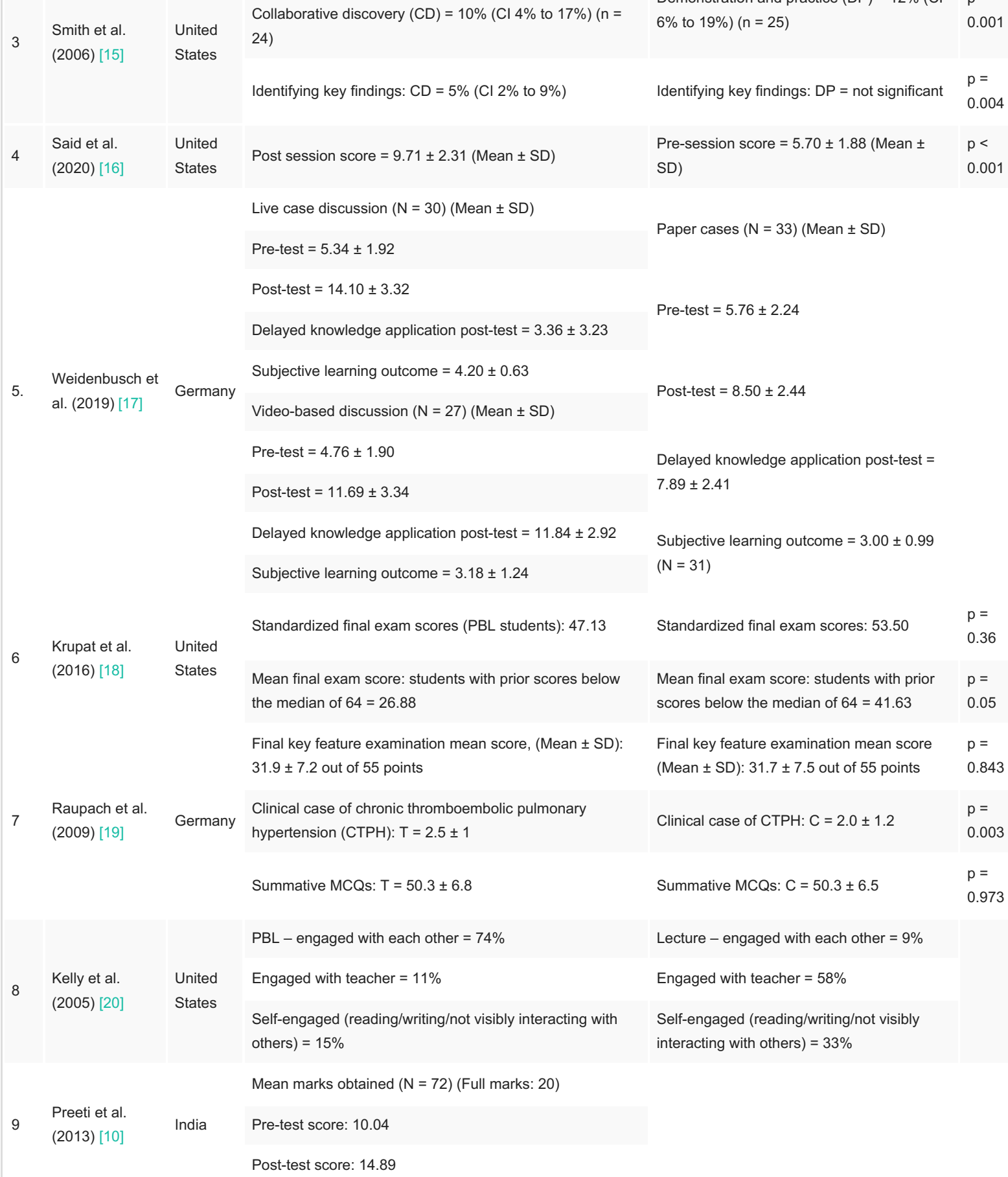

\section{TABLE 1: Summary of various studies regarding problem-based learning.}

$\mathrm{PBL}=$ problem-based learning; $\mathrm{TBL}=$ team-based learning; $\mathrm{E}-\mathrm{PBL}=$ electronic problem-based learning; $\mathrm{CKD}=$ chronic kidney disease; $\mathrm{CD}=\mathrm{Crohn}$ 's disease; $\mathrm{MCQ}=$ multiple choice question; $\mathrm{UTI}=$ urinary tract infection; $\mathrm{SD}=$ standard deviation.

Interpersonal Communication

We included two studies under this heading. The first study was conducted in Switzerland to assess the triggers for conflict in a team and its impact on teamwork. Team members perceived that observed tensions were directly related to lower quality work based on multilevel regression analysis (except among anesthetists) with or without adjusting for hospital and surgery duration. Moreover, the quality of teamwork was rated high by all surgical team members [21]. The second study in Singapore showed increased selfconfidence among the healthcare workers when they underwent a simulation-based interprofessional educational program for caring for a deteriorating patient [22]. Subsequently, a meta-analysis by Zolnierek 


\section{Cureus}

et al. revealed marked improvement in patient adherence after the physician communication training. Furthermore, it was clearly stated that non-adherence was common among the patients attended by physicians who communicate poorly [23]. All these findings highlight the importance of interpersonal communication.

Summary of the studies done regarding interpersonal communication is presented in Table $2[21,24]$.

\begin{tabular}{|c|c|c|c|c|c|}
\hline No. & Study ID & Country & Interpersonal communication & Controls & $\begin{array}{l}\text { p- } \\
\text { value }\end{array}$ \\
\hline \multirow{12}{*}{1} & \multirow{12}{*}{$\begin{array}{l}\text { Keller et al. } \\
\text { (2019) [21] }\end{array}$} & \multirow{12}{*}{ Switzerland } & Total surgeries observed $(\mathrm{N}=137)$ & \multirow{8}{*}{$\begin{array}{l}\text { Tension-free surgeon heading a surgical } \\
\text { team }=12\end{array}$} & \\
\hline & & & Hospital 1 (H1) N1 = 86 & & \\
\hline & & & Hospital $2(\mathrm{H} 2) \mathrm{N} 2=51$ & & \\
\hline & & & $\begin{array}{l}\text { Mean duration between incision and closure: (mean } \pm \\
\text { standard deviation) }=3.67 \pm 2.21 \text { hours }\end{array}$ & & \\
\hline & & & $\mathrm{H} 1=3.74 \pm 2.43$ hours & & \\
\hline & & & $\mathrm{H} 2=3.55 \pm 1.89$ hours & & \\
\hline & & & Total tense communications $=340$ & & \\
\hline & & & $\begin{array}{l}\text { Tensions per surgery (mean } \pm \text { standard deviation })=2.48 \pm \\
5.19\end{array}$ & & \\
\hline & & & $\begin{array}{l}\text { Tensions per hour of surgery (mean } \pm \text { standard deviation })= \\
0.57 \pm 1.02\end{array}$ & & \\
\hline & & & Tension free surgeries $=72(52.6 \%)$ & & \\
\hline & & & Total surgeons $=30$ & & \\
\hline & & & Teamwork quality with tension $=4.87$ on a 7 -point scale & $\begin{array}{l}\text { Teamwork quality without tension }=6.20 \\
\text { on a } 7 \text {-point scale }\end{array}$ & \\
\hline \multirow{3}{*}{2} & \multirow{3}{*}{$\begin{array}{l}\text { Liaw et al. } \\
\text { (2014) [24] }\end{array}$} & \multirow{3}{*}{ Singapore } & $\begin{array}{l}\text { Medical students (N1 = 33), nursing students }(\mathrm{N} 2=92) \text {, } \\
\text { medical and nursing group }\end{array}$ & & \\
\hline & & & $\begin{array}{l}\text { Selt-contidence: Scale: } 5 \text {-50, mean } \pm \text { standard deviatıon = } \\
34.26 \pm 6.00\end{array}$ & mean \pm standard deviation $=27.68 \pm 6.42$ & $\begin{array}{l}p= \\
0.001\end{array}$ \\
\hline & & & $\begin{array}{l}\text { Perception: Scale: } 8-40, \text { mean } \pm \text { standard deviation }=36.36 \\
\pm 3.46\end{array}$ & $\begin{array}{l}\text { Perception: Scale: } 8-40, \text { mean } \pm \text { standard } \\
\text { deviation }=31.90 \pm 3.84\end{array}$ & $\begin{array}{l}p= \\
0.001\end{array}$ \\
\hline
\end{tabular}

TABLE 2: Studies regarding interpersonal communication.

Patient Care

Three studies in our review showed improved patient satisfaction and care after a collaborative and multimodal approach in their management $[5,25,26]$.

A study in the United States of America that focused on improving the quality of diabetes care in community centers was intervened by collaboration with community organizations, a self-management tool to track the patient's progress, group cluster visits, and diabetes flow sheet. Of the respondents, 95\% strongly believed that the collaboration was successful, and $>80 \%$ wished to continue the interventions [25]. Similarly, a study in Sweden using a multi-modality approach to pain management for musculoskeletal pain found a significant improvement in patients' post-intervention status, which was sustained at the 12 months. However, the longer duration of the program was not found to be beneficial compared to shorter-duration programs [5]. A systematic review done by Hush et al. also exhibited that patient satisfaction is determined by interpersonal attributes and patient care approach. However, patient satisfaction was inconsistent with the treatment outcome [27]. Table 3 summarizes the findings of included studies. 


\section{Cureus} $\begin{array}{ll}\text { 1. Chin et al. } & \text { United } \\ \text { (2004) [25] } & \text { States }\end{array}$

\begin{tabular}{|c|c|c|c|c|c|}
\hline \multirow{20}{*}{1.} & \multirow{20}{*}{$\begin{array}{l}\text { Chin et al. } \\
(2004)[25]\end{array}$} & \multirow{20}{*}{$\begin{array}{l}\text { United } \\
\text { States }\end{array}$} & Matched patients $(n=969)$ & & \\
\hline & & & One HbA1c measurement: 1998: $80 \%$ & 1999: $90 \%$ & $<0.001$ \\
\hline & & & Two HbA1c measurements at least 3 months apart: 1998: $37 \%$ & 1999: $54 \%$ & $<0.001$ \\
\hline & & & Eye exam referral: 1998: $36 \%$ & 1999: $47 \%$ & 0.02 \\
\hline & & & Dietary counseling/referral to nutritionist: $1998: 51 \%$ & 1999: $57 \%$ & 0.10 \\
\hline & & & Foot exam/referral to podiatrist: $1998: 40 \%$ & 1999: 64\% & $<0.001$ \\
\hline & & & Dental referral: 1998: 7.2\% & 1999: $18 \%$ & 0.001 \\
\hline & & & Lipid assessment: 1998: 55\% & 1999: $66 \%$ & 0.02 \\
\hline & & & Urine microalbumin assessment: 1998: $13 \%$ & 1999: $25 \%$ & 0.001 \\
\hline & & & HbA1c value (\%): 1998: 8.51\% & 1999: 8.32\% & 0.09 \\
\hline & & & All patients $(n=1628)$ & & \\
\hline & & & One HbA1c measurement: 1998: 80\% & 1999: $90 \%$ & $<0.001$ \\
\hline & & & Two HbA1c measurements at least 3 months apart: 1998: $38 \%$ & 1999: $54 \%$ & $<0.001$ \\
\hline & & & Eye exam referral: 1998: 36\% & 1999: $47 \%$ & 0.02 \\
\hline & & & Dietary counseling/referral to nutritionist: $1998: 49 \%$ & 1999: $58 \%$ & 0.04 \\
\hline & & & Foot exam/referral to podiatrist: $1998: 40 \%$ & 1999: $64 \%$ & $<0.001$ \\
\hline & & & Dental referral: 1998: 6.7\% & 1999: $17 \%$ & $<0.001$ \\
\hline & & & Lipid assessment: 1998: 55\% & 1999: $67 \%$ & 0.005 \\
\hline & & & Urine microalbumin assessment: 1998: $12 \%$ & 1999: $25 \%$ & 0.001 \\
\hline & & & HbA1c value (\%): 1998: 8.52\% & 1999: $8.40 \%$ & 0.30 \\
\hline \multirow{18}{*}{2.} & \multirow{18}{*}{$\begin{array}{l}\text { Tseli et al. } \\
(2020)[5]\end{array}$} & \multirow{18}{*}{ Sweden } & $\begin{array}{l}\text { Short-form Health Survey (SF-36) Physical Component } \\
\text { Summary (PCS) (0-100) }\end{array}$ & $\begin{array}{l}\text { Post-Interdisciplinary Multimodel Pain } \\
\text { Rehabilitation (Post IMPR) }\end{array}$ & \\
\hline & & & Short: Baseline 27.9 & Post IMPR 30.2 & \\
\hline & & & Medium: Baseline 28.6 & Post IMPR 31.6 & $<0.001$ \\
\hline & & & Long: Baseline 29.3 & Post IMPR 29.7 & 0.335 \\
\hline & & & SF-36 Mental Component Summary (MCS) (0-100) & & \\
\hline & & & Short: Baseline 36.2 & Post IMPR 39.6 & \\
\hline & & & Medium: Baseline 33.8 & Post IMPR 38.6 & 0.084 \\
\hline & & & Long: Baseline 33.7 & Post IMPR 39.5 & 0.853 \\
\hline & & & Pain intensity last 7 days & & \\
\hline & & & Short: Baseline 7.0 & Post IMPR 6.2 & \\
\hline & & & Medium: Baseline 7.0 & Post IMPR 6.1 & 0.166 \\
\hline & & & Long: Baseline 6.8 & Post IMPR 6.2 & 0.891 \\
\hline & & & Euro-Qol 5-dimensions (EQ-5D index) (-0.594-1) & & \\
\hline & & & Short: Baseline 0.25 & Post IMPR 0.35 & \\
\hline & & & Medium: Baseline 0.22 & Post IMPR 0.37 & 0.313 \\
\hline & & & Long: Baseline 0.28 & Post IMPR 0.34 & 0.632 \\
\hline & & & Hospital Anxiety and Depression Scale (HADS) A (0-21) & & \\
\hline & & & Short: Baseline 8.8 & Post IMPR 7.9 & \\
\hline
\end{tabular}




\section{Cureus}

\begin{tabular}{|c|c|c|c|c|c|}
\hline & & & Long: Baseline 29.3 & \multirow[t]{2}{*}{ Post IMPR 29.7} & \multirow[t]{2}{*}{0.941} \\
\hline & & & HADS A (0-21) & & \\
\hline & & & Short: Baseline 8.5 & \multicolumn{2}{|l|}{ Post IMPR 6.5} \\
\hline & & & Medium: Baseline 9.0 & Post IMPR 6.6 & 0.519 \\
\hline & & & Long: Baseline 8.9 & Post IMPR 6.7 & 0.293 \\
\hline \multirow{2}{*}{3.} & \multirow{2}{*}{$\begin{array}{l}\text { Schiff et al. } \\
(2017)[26]\end{array}$} & \multirow{2}{*}{$\begin{array}{l}\text { United } \\
\text { States }\end{array}$} & $\begin{array}{l}\text { Rates of potential patient safety risks: Pre-intervention: } 155 \text { per } \\
1000 \text { patients with an abnormal lab value }\end{array}$ & $\begin{array}{l}\text { Post-intervention: } 54 \text { per } 1000 \text { patients } \\
\text { with abnormal lab value }\end{array}$ & $\begin{array}{l}(95 \% \mathrm{Cl} \\
0.24-0.50)\end{array}$ \\
\hline & & & $\begin{array}{l}\text { Rates of serious patient safety risks: Pre-intervention: } 28 \text { per } \\
1000 \text { patients with an abnormal lab value }\end{array}$ & $\begin{array}{l}\text { Post-intervention: } 13 \text { per } 1000 \text { patients } \\
\text { with abnormal lab value }\end{array}$ & $\begin{array}{l}(95 \% \mathrm{Cl} \\
0.22-0.98)\end{array}$ \\
\hline
\end{tabular}

TABLE 3: Summary of various studies included under "patient care."

$\mathrm{HbA1 \textrm {c }}=$ glycosylated hemoglobin .

Professionalism

A total of five studies qualified for the study under the heading of "professionalism" and are summarized in Table 4 [28-31]. Most health professionals are well aware of professionalism and its impact on medical education and clinical practice [31]. In a study in Japan, emergency medicine (EM) residents scored higher than EM physicians when questioned about confidentiality and sexual harassment [32]. Another study showed that clinical groups of students scored higher than preclinical students in a quiz about professionalism. However, the finding was not statistically significant [29]. In a study done in Australia, 95\% of respondents stated that personal and professional development (PPD) helped them learn about professional development. Interviewing patients in the community and writing from the patient's perspective helped students understand the biopsychosocial aspect of medicine and guided appropriate behavior in clinical practice [6]. Health professionals who are well informed about work habits and work ethics will foster patient adherence and create a healthy working environment.

\begin{tabular}{|c|c|c|c|c|c|}
\hline $\begin{array}{l}\text { S. } \\
\text { No. }\end{array}$ & Year & Country & Outcome & & $\begin{array}{l}\mathrm{p}- \\
\text { value }\end{array}$ \\
\hline \multirow{14}{*}{1.} & \multirow{12}{*}{$\begin{array}{l}\text { Wiecha et al. } \\
(2008)[28]\end{array}$} & \multirow{12}{*}{$\begin{array}{l}\text { United } \\
\text { States }\end{array}$} & $\begin{array}{l}\text { Identifying factors contributing to patient non-compliance control: number } \\
\text { (\%) }\end{array}$ & $\begin{array}{l}\text { Intervention group: } \\
\text { number }(\%)\end{array}$ & \\
\hline & & & Decreased 9 (8.8\%) & Decreased 5 (4.5\%) & \\
\hline & & & No change 43 (42.2\%) & No change 29 (25.9\%) & \\
\hline & & & Gained 50 (49.0\%) & Gained $78(69.6 \%)$ & \\
\hline & & & $\begin{array}{l}\text { Integrating patient's cultural beliefs about health into your care of that } \\
\text { patient control: number (percentage) }\end{array}$ & $\begin{array}{l}\text { Intervention group: } \\
\text { number (percentage) }\end{array}$ & \\
\hline & & & Decreased $11(10.7 \%)$ & Decreased 6 (5.4\%) & \\
\hline & & & No change 41 (39.8\%) & No change 25 (22.3\%) & \\
\hline & & & Gained 51 (49.5\%) & Gained $81(72.3 \%)$ & \\
\hline & & & $\begin{array}{l}\text { Eliciting how a patient has been emotionally impacted by an illness } \\
\text { control: number (percentage) }\end{array}$ & $\begin{array}{l}\text { Intervention group: } \\
\text { number (percentage) }\end{array}$ & \\
\hline & & & Decreased $14(13.5 \%)$ & Decreased 8 (7.1\%) & \\
\hline & & & No change 42 (40.4\%) & No change $28(25.0 \%)$ & \\
\hline & & & Gained $48(46.2 \%)$ & Gained $76(67.9 \%)$ & \\
\hline & & & $\begin{array}{l}\text { Frequency of the best or second-best responses for scenarios presented } \\
\text { by residents and faculty: number (percentage) }\end{array}$ & Number (percentage) & \\
\hline & & & Gifts: Total $(\mathrm{T})=153$ (88.9\%); Resident = $73(87.9 \%)$ & Faculty = 80 (89.8\%) & 0.81 \\
\hline
\end{tabular}




\section{Cureus}

Shiga et al. (2020) Japan
[32]

Haque et al. (2016) [29]

5

Curran et al. (2020)

[30]

Byakika-Kibwika et al. (2015) [31]
Conflict of interest: $\mathrm{T}=154(89.7 \%)$; Resident $=72(86.7 \%)$

Faculty $=82(92.2 \%)$

0.32

Confidentiality: $\mathrm{T}=121(69.9 \%)$; Resident $=64$ (77.1\%)

Faculty $=56(62.9 \%) \quad 0.048$

Impairment: $\mathrm{T}=145(84.3 \%) ;$ Resident $=67(80.7 \%)$

Faculty $=78(87.6 \%)$

0.29

Harassment: $\mathrm{T}=77$ (44.5\%); Resident $=36$ (43.3\%)

Faculty $=41(46.1 \%) \quad 0.76$

Honesty: $\mathrm{T}=151$ (87.3\%); Resident $=76(81.7 \%)$

Faculty $=74(83.1 \%) \quad 0.11$

Professionalism according to sex: mean (SD)

Honesty: Male: 22.39 (3.69)

Female: $22.33(3.26) \quad 0.956$

Accountability: Male: $17.82(2.70)$

Female:18.69(3.12) $\quad 0.72$

Confidentiality: Male 15.31 (2.28)

Female: $15.61(3.42) \quad 0.531$

Respectful: Male 24.00 (3.09)

Responsibility: Male 22.35 (2.99)

Female: 24.76(3.14) $\quad 0.190$

Female 23.58(2.82) $\quad 0.020$

Compassion: Male 15.76 (2.64)

Female 16.99(5.98) $\quad 0.130$

Communication: Male 18.06 (3.28)

Female 19.10(3.00) $\quad 0.055$

Maturity: Male 23.39 (3.66)

Female $24.04(2.85) \quad 0.248$

Self-directed learning: Male 7.88 (1.29)

Female $8.24(1.51) \quad 0.086$

Grand total score: Male 166.98 (20.15)

Female 173.34 (18.09)

0.61

Comparison of mean score of professionalism by medical students according to educational phase: mean (SD) total possible score: 220

Honesty: Preclinical: 22.67 (3.52)

Clinical: $22.11(3.34) \quad 0.361$

Accountability: Preclinical: 18.02 (2.48)

Clinical: 18.62(3.31) $\quad 0.195$

Confidentiality: Preclinical 15.44 (3.47)

Clinical: $15.54(2.67) \quad 0.762$

Respectful: Preclinical 24.19 (3.25)

Clinical: $24.68(3.04) \quad 0.234$

Responsibility: Preclinical 23.33 (2.82)

Clinical:23.14(3.04) $\quad 0.676$

Compassion: Preclinical 16.36 (2.50)

Clinical: $16.64(6.29) \quad 0.733$

Communication: Preclinical 18.36 (3.39)

Clinical: $18.97(2.93) \quad 0.179$

Maturity: Preclinical 23.01 (3.42)

Clinical $23.70(3.01) \quad 0.918$

Self-directed learning: Preclinical 8.15 (1.41)

Clinical: $8.12(1.45) \quad 0.923$

Grand total score: Preclinical 170.17 (18.67)

Clinical: $171.49(19.49) \quad 0.694$

Male: $166.98 \pm 20.15$

Female: $173.34+/-18.09 \quad$ NS

Preclinical: $170.17 \pm 18.67$

Clinical: $171.49+/-19.49 \quad$ NS

Friedman analysis of peer assessment score improvement over time; Work Habits Score (TP = time point)

Mean: TP1 26.25; TP2 26.5; TP3 26.93; TP4 27.45; Friedman X2 (3) $=52.07$

Mean Rank: TP1 1.95; TP2 2.22; TP3 2.50; TP4 3.33

Interpersonal Habits Score

Mean: TP1 28.19; TP2 28.56; TP3 28.83 TP4 29.05; Friedman X2(3) $=56.23$

Mean Rank TP1 1.73; TP2 2.38; TP3 2.75; TP4 3.15

Duration in teaching service: median: 39; IQR 32.7-43.2

Doctorate degree: 7 (22\%)

Master's degree: 23 (72\%); Fellowship: $1(3 \%)$

Bachelor's degree 1 (3\%) 


\section{Cureus}

TABLE 4: Summary of various studies regarding professionalism.

$I Q R=$ interquartile range

System-Based Care

Multiple studies have shown that system-based care aligns with the quality of patient care and decreases the risks associated with the clinical practice $[8,33]$. In addition, inter-specialty collaboration to manage hard and soft tissue injuries with a systematic approach has also boosted the confidence and reinforced their surgical skills [34]. For example, a study undertaken in the United States implementing the Six Building Blocks Program for managing patients taking opioids for chronic pain analyzed its effect on the work-life of primary care providers and staff. As a result, the involved staff reported improved work-life balance, confidence and comfort in clinical areas, ease in managing cases with chronic pain, increased comfort in work processes and their role, and increased collaboration after implementing the program [33]. Thus, system-based care has a two-way advantage where the patient gets better care along with physician satisfaction. Table 5 summarizes the study findings.

\begin{tabular}{|c|c|c|c|c|}
\hline $\begin{array}{l}\text { No. } \\
\text { No }\end{array}$ & Year & Country & Outcome & \\
\hline \multirow{4}{*}{1.} & \multirow{4}{*}{$\begin{array}{l}\text { Milne et al. } \\
(2020)[34]\end{array}$} & \multirow{4}{*}{$\begin{array}{l}\text { United } \\
\text { Kingdom }\end{array}$} & \multirow{2}{*}{ Before course: } & $\begin{array}{l}\text { Reasons for inability to treat facial and oral wounds in } \\
\text { the emergency department: }\end{array}$ \\
\hline & & & & Service pressures \\
\hline & & & $\begin{array}{l}25 / 48 \text { participants: confident to repair lip laceration in an } \\
\text { adult either with supervision or independently }\end{array}$ & Lack of confidence in the ability \\
\hline & & & $\begin{array}{l}15 / 48 \text { participants: confident to repair full-thickness } \\
\text { laceration of the pinna }\end{array}$ & $\begin{array}{l}\text { Inability to provide adequate analgesia (including } \\
\text { sedating children) }\end{array}$ \\
\hline \multirow{7}{*}{2.} & \multirow{7}{*}{$\begin{array}{l}\text { Stevens et } \\
\text { al. (2010) [8] }\end{array}$} & \multirow{7}{*}{$\begin{array}{l}\text { United } \\
\text { States }\end{array}$} & $\begin{array}{l}\text { Clinical outcomes for } 15 \text { teams in California Academic } \\
\text { Chronic Care Collaborative June } 2007\end{array}$ & $\begin{array}{l}\text { Clinical outcomes for } 15 \text { teams in California Academic } \\
\text { Chronic Care Collaborative May } 2008\end{array}$ \\
\hline & & & $\begin{array}{l}\text { HbA1c }<7 \% \text {, total registry size }(n=1302) \text {; weighted } \\
\text { average } 42.4\end{array}$ & $\begin{array}{l}\text { HbA1c }<7 \% \text {, total registry size }(n=1559) \text {; weighted } \\
\text { average } 44.7\end{array}$ \\
\hline & & & $\begin{array}{l}\mathrm{LDL}<100 \mathrm{mg} / \mathrm{dL} \text {, total registry size }(\mathrm{n}=1034) \text {; weighted } \\
\text { average } 50.9\end{array}$ & $\begin{array}{l}\mathrm{LDL}<100 \mathrm{mg} / \mathrm{dL} \text {, total registry size }(\mathrm{n}=1351) \\
\text { weighted average } 59.5\end{array}$ \\
\hline & & & $\begin{array}{l}\text { BP }<130 / 80 \text {, total registry size }(n=1302) \text {; weighted } \\
\text { average } 36.4\end{array}$ & $\begin{array}{l}\text { BP }<130 / 80 \text { total registry size }(n=1559) ; \text { weighted } \\
\text { average } 47.4\end{array}$ \\
\hline & & & $\begin{array}{l}\text { Retinal exam total registry size }(n=1178) \text {; weighted } \\
\text { average } 25.5\end{array}$ & $\begin{array}{l}\text { Retinal exam total registry size }(n=1437) \text {; weighted } \\
\text { average } 41.1\end{array}$ \\
\hline & & & $\begin{array}{l}\text { Foot exam total registry size }(n=1178) \text {; weighted average } \\
30.4\end{array}$ & $\begin{array}{l}\text { Foot exam total registry size }(n=1437) \text {; weighted } \\
\text { average } 56\end{array}$ \\
\hline & & & $\begin{array}{l}\text { Documented self-management goal total registry size }(n= \\
1300) ; \text { weighted average } 10.7\end{array}$ & $\begin{array}{l}\text { Documented self-management goal total registry size } \\
\text { ( } n=1559) \text {; weighted average } 41.4\end{array}$ \\
\hline
\end{tabular}

TABLE 5: Summary of studies regarding system-based care.

$\mathrm{HbA1c}=$ glycosylated hemoglobin; $\mathrm{LDL}=$ low-density lipoprotein; $\mathrm{BP}=$ blood pressure .

Practice-Based Learning and Improvement

Practice-based learning and improvement (PBLI) connect continuous learning to good patient care. It is an experiential continuum that reveals trainees their own learning needs and the needs of their practices. The trainees then develop and implement plans for their self-improvement and the improvement of their practices. Small and sustained changes in individual clinicians and practice patterns can result in the improvement of healthcare systems [35]. For example, a study by Ogrinc et al. in 2004 revealed that four 
weeks of PBLI elective by internal medicine residents improved quality Improvement Knowledge Application Tool scores compared to the control group [36]. Other studies by Varkey et al. in 2009 describe that the application of PBLI and systems-based practice in the curriculum in Mayo Clinic resulted in a 13\% increase in perceived ability to measure competency in systolic blood pressure (SBP), no change in their perceived ability to measure competence in PBLI, a $15 \%$ increase in their ability to provide written documentation of competence in PBLI and a 35\% increase in their ability to provide written documentation of competence in SBP between 2005 and 2007. In addition, 70\% of the residents participated in quality improvement (QI) projects during the time [37]. Therefore, it is crucial to develop and implement a curriculum in PBLI by every teaching medical institution.

\section{Conclusions}

Improvement of medical education involves integrating problem-based learning, robust interpersonal communication, patient care, professionalism, and practice-based learning and improvement and improving system-based care. Incorporating and implementing these core competencies in medical curricula is essential in all levels of medical training, including undergraduate level, residency, and fellowship training.

\section{Additional Information \\ Disclosures}

Conflicts of interest: In compliance with the ICMJE uniform disclosure form, all authors declare the following: Payment/services info: All authors have declared that no financial support was received from any organization for the submitted work. Financial relationships: All authors have declared that they have no financial relationships at present or within the previous three years with any organizations that might have an interest in the submitted work. Other relationships: All authors have declared that there are no other relationships or activities that could appear to have influenced the submitted work.

\section{References}

1. Patel M: Changes to postgraduate medical education in the 21st century . Clin Med (Lond). 2016, 16:311-4. 10.7861/clinmedicine.16-4-311

2. Edgar L, McLean S, Hogan SO, Hamstra S, Holmboe ES: The Milestones Guidebook. Accreditation Council for Graduate Medical Education, Chicago, Illinois; 2020.

3. Karimi R: Interface between problem-based learning and a learner-centered paradigm . Adv Med Educ Pract. 2011, 2:117-25. 10.2147/AMEP.S12794

4. Boshoff K, Murray C, Worley A, Berndt A: Interprofessional education placements in allied health: a scoping review. Scand J Occup Ther. 2020, 27:80-97. 10.1080/11038128.2019.1642955

5. Tseli E, LoMartire R, Vixner L, Grooten WJ, Gerdle B, Äng BO: What Is the effectiveness of different duration interdisciplinary treatment programs in patients with chronic pain? A large-scale longitudinal register study. J Clin Med. 2020, 9:2788. 10.3390/jcm9092788

6. Langendyk V, Mason G, Wang S: How do medical educators design a curriculum that facilitates student learning about professionalism?. Int J Med Educ. 2016, 7:32-43. 10.5116/ijme.5683.c2e0

7. Reimer D, Russell R, Khallouq BB, Kauffman C, Hernandez C, Cendán J, Castiglioni A: Pre-clerkship medical students' perceptions of medical professionalism. BMC Med Educ. 2019, 19:239. 10.1186/s12909-019-1629-4

8. Stevens DP, Bowen JL, Johnson JK, et al.: A multi-institutional quality improvement initiative to transform education for chronic illness care in resident continuity practices. J Gen Intern Med. 2010, 25:574-80. 10.1007/s11606-010-1392-z

9. Dressler DD, Pistoria MJ, Budnitz TL, McKean SC, Amin AN: Core competencies in hospital medicine: development and methodology. J Hosp Med. 2006, 1:48-56. 10.1002/jhm.6

10. Preeti B, Ashish A, Shriram G: Problem based learning (PBL) - an effective approach to improve learning outcomes in medical teaching. J Clin Diagn Res. 2013, 7:2896-7. 10.7860/JCDR/2013/7339.3787

11. Qin Y, Wang Y, Floden RE: The effect of problem-based learning on improvement of the medical educational environment: a systematic review and meta-analysis. Med Princ Pract. 2016, 25:525-32. $10.1159 / 000449036$

12. Galvao TF, Silva MT, Neiva CS, Ribeiro LM, Pereira MG: Problem-based learning in pharmaceutical education: a systematic review and meta-analysis. ScientificWorldJournal. 2014, 2014:578382. $10.1155 / 2014 / 578382$

13. Robson J: Web-based learning strategies in combination with published guidelines to change practice of primary care professionals. Br J Gen Pract. 2009, 59:104-9. 10.3399/bjgp09X395102

14. Alaagib NA, Musa OA, Saeed AM: Comparison of the effectiveness of lectures based on problems and traditional lectures in physiology teaching in Sudan. BMC Med Educ. 2019, 19:365. 10.1186/s12909-0191799-0

15. Smith CA, Hart AS, Sadowski LS, et al.: Teaching cardiac examination skills. A controlled trial of two methods. J Gen Intern Med. 2006, 21:7-12. 10.1111/j.1525-1497.2005.00254.x

16. Said JT, Thompson LL, Foord L, Chen ST: Impact of a case-based collaborative learning curriculum on knowledge and learning preferences of dermatology residents. Int J Womens Dermatol. 2020, 6:404-8. 10.1016/j.ijwd.2020.06.002

17. Weidenbusch M, Lenzer B, Sailer M, et al.: Can clinical case discussions foster clinical reasoning skills in undergraduate medical education? A randomised controlled trial. BMJ Open. 2019, 9:e025973. 10.1136/bmjopen-2018-025973

18. Krupat E, Richards JB, Sullivan AM, Fleenor TJ Jr, Schwartzstein RM: Assessing the effectiveness of casebased collaborative learning via randomized controlled trial. Acad Med. 2016, 91:723-9. 
10.1097/ACM.0000000000001004

19. Raupach T, Muenscher C, Anders S, Steinbach R, Pukrop T, Hege I, Tullius M: Web-based collaborative training of clinical reasoning: a randomized trial. Med Teach. 2009, 31:e431-7. 10.1080/01421590903095502

20. Kelly PA, Haidet P, Schneider V, Searle N, Seidel CL, Richards BF: A comparison of in-class learner engagement across lecture, problem-based learning, and team learning using the STROBE classroom observation tool. Teach Learn Med. 2005, 17:112-8. 10.1207/s15328015tlm1702_4

21. Keller S, Tschan F, Semmer NK, et al.: "Disruptive behavior" in the operating room: a prospective observational study of triggers and effects of tense communication episodes in surgical teams. PLoS One. 2019, 14:e0226437. 10.1371/journal.pone.0226437

22. Liaw SY, Zhou WT, Lau TC, Siau C, Chan SW: An interprofessional communication training using simulation to enhance safe care for a deteriorating patient. Nurse Educ Today. 2014, 34:259-64. 10.1016/j.nedt.2013.02.019

23. Zolnierek KB, Dimatteo MR: Physician communication and patient adherence to treatment: a metaanalysis. Med Care. 2009, 47:826-34. 10.1097/MLR.0b013e31819a5acc

24. Liaw SY, Ooi SW, Rusli KD, Lau TC, Tam WW, Chua WL: Nurse-physician communication team training in virtual reality versus live simulations: randomized controlled trial on team communication and teamwork attitudes. J Med Internet Res. 2020, 22:e17279. 10.2196/17279

25. Chin MH, Cook S, Drum ML, et al.: Improving diabetes care in Midwest community health centers with the health disparities collaborative. Diabetes Care. 2004, 27:2-8. 10.2337/diacare.27.1.2

26. Schiff GD, Reyes Nieva H, Griswold P, et al.: Randomized trial of reducing ambulatory malpractice and safety risk: results of the Massachusetts PROMISES project. Med Care. 2017, 55:797-805. 10.1097/MLR.0000000000000759

27. Hush JM, Cameron K, Mackey M: Patient satisfaction with musculoskeletal physical therapy care: a systematic review. Phys Ther. 2011, 91:25-36. 10.2522/ptj.20100061

28. Wiecha JM, Markuns JF: Promoting medical humanism: design and evaluation of an online curriculum. Fam Med. 2008, 40:617-9.

29. Haque M, Zulkifli Z, Haque SZ, et al.: Professionalism perspectives among medical students of a novel medical graduate school in Malaysia. Adv Med Educ Pract. 2016, 7:407-22. 10.2147/AMEP.S90737

30. Curran VR, Sharpe D, Forristall J, Flynn K: Student satisfaction and perceptions of small group process in case-based interprofessional learning. Med Teach. 2008, 30:431-3. 10.1080/01421590802047323

31. Byakika-Kibwika P, Kutesa A, Baingana R, et al.: A situation analysis of inter-professional education and practice for ethics and professionalism training at Makerere University College of Health Sciences. BMC Res Notes. 2015, 8:598. 10.1186/s13104-015-1577-y

32. Shiga T, Nakashima Y, Norisue Y, et al.: Comparison of professionalism between emergency medicine resident physicians and faculty physicians: a multicenter cross-sectional study. PLoS One. 2020, 15:e0230186. 10.1371/journal.pone.0230186

33. Ike B, Baldwin LM, Sutton S, Van Borkulo N, Packer C, Parchman ML: Staff and clinician work-life perceptions after implementing systems-based improvements to opioid management. J Am Board Fam Med. 2019, 32:715-23. 10.3122/jabfm.2019.05.190027

34. Milne S, Walshaw EG, Webster A, Mannion CJ: Active learning in head and neck trauma: outcomes after an innovative educational course. [PREPRINT]. Br J Oral Maxillofac Surg. 2020, 10.1016/j.bjoms.2020.05.003

35. Lynch DC, Swing SR, Horowitz SD, Holt K, Messer JV: Assessing practice-based learning and improvement . Teach Learn Med. 2004, 16:85-92. 10.1207/s15328015tlm1601_17

36. Ogrinc G, Headrick LA, Morrison LJ, Foster T: Teaching and assessing resident competence in practice-based learning and improvement. J Gen Intern Med. 2004, 19:496-500. 10.1111/j.1525-1497.2004.30102.x

37. Varkey P, Karlapudi S, Rose S, Nelson R, Warner M: A systems approach for implementing practice-based learning and improvement and systems-based practice in graduate medical education. Acad Med. 2009, 84:335-9. 10.1097/ACM.0b013e31819731fb 